\title{
Training in diagnostic radiology
}

Jan Lotz, MA, MSc, MMed, FCRad, FRCR

Department of Radiology, Stellenbosch University
Training in diagnostic radiology has been debated extensively in this country. When I started as a registrar in 1975, diagnostic radiology was limited to 'black and white' and fluoroscopy. The academic curriculum was covered in a 4-year course, the first $12-18$ months being devoted to acquiring the primary qualifications in anatomy, physics and physiology in most of the MMed courses.

Since the mid-70s we have seen a digital imaging explosion. First 'realtime' ultrasound took centre stage, followed by computed tomography (CT), digital subtraction angiography, magnetic resonance imaging (MRI), interventional radiology and PACS. In the early nineties it seemed as if we had exhausted all technical development with real-time three-dimensional imaging, then the second tsunami hit. This time we saw multidetector CT with coronary imaging, real-time MR imaging of the heart, MR spectroscopy, diffusion and perfusion imaging, functional MRI and whole-body CT data collection.

At the time of writing in 2006 and despite the earth-shattering developments listed above, it is with deep regret that I have to report that diagnostic radiology remains a 4-year course, of which the first 12 - 18 months are still devoted to passing the primary examinations.

Inertia to change over the past two and a half decades has been caused by many factors. In a recent editorial in the SAJR Prof Coert de Vries alluded to chronic problems in our academic hospitals that prevented necessary adaptations to our concepts of training and research.

This is water under the bridge. In a recent report on the current status of radiologist training in South Africa, conducted at the 1st and 2nd preexamination courses of the College of Radiology of South Africa, the majority ( $82.3 \%$ and $91.5 \%$, respectively) of the attendees said that they received 'formal' lectures and tutorials. The majority (72.3\%) of the respondents on course 2 felt that both their training and the structuring of training were adequate. Likewise, they felt that their hospital equipment was adequate $(57.4 \%)$. In contrast, fewer of the respondents on course 1 felt the same way - only $47 \%$ of the respondents felt that their training and structuring of their training were adequate and only $36.7 \%$ felt that their hospital equipment was adequate. Less than half of the respondents on both courses said that they received formal assessments . Of the candidates, $75 \%$ and $85.1 \%$ on course 1 and course 2, respectively, intended to remain in a registrar post for a 5 th year in departments where this was possible.

Professor Savvas Andronikou will present this report and some of our suggestions at a special session on education and training at the ISR Congress in September. We believe that an integrated National Curriculum should be tabled. Tuition should be structured accordingly and study material shared. Table I represents an abstract of a 4-year modular course that was introduced at the University of the Free State in 2004. Registrars are 


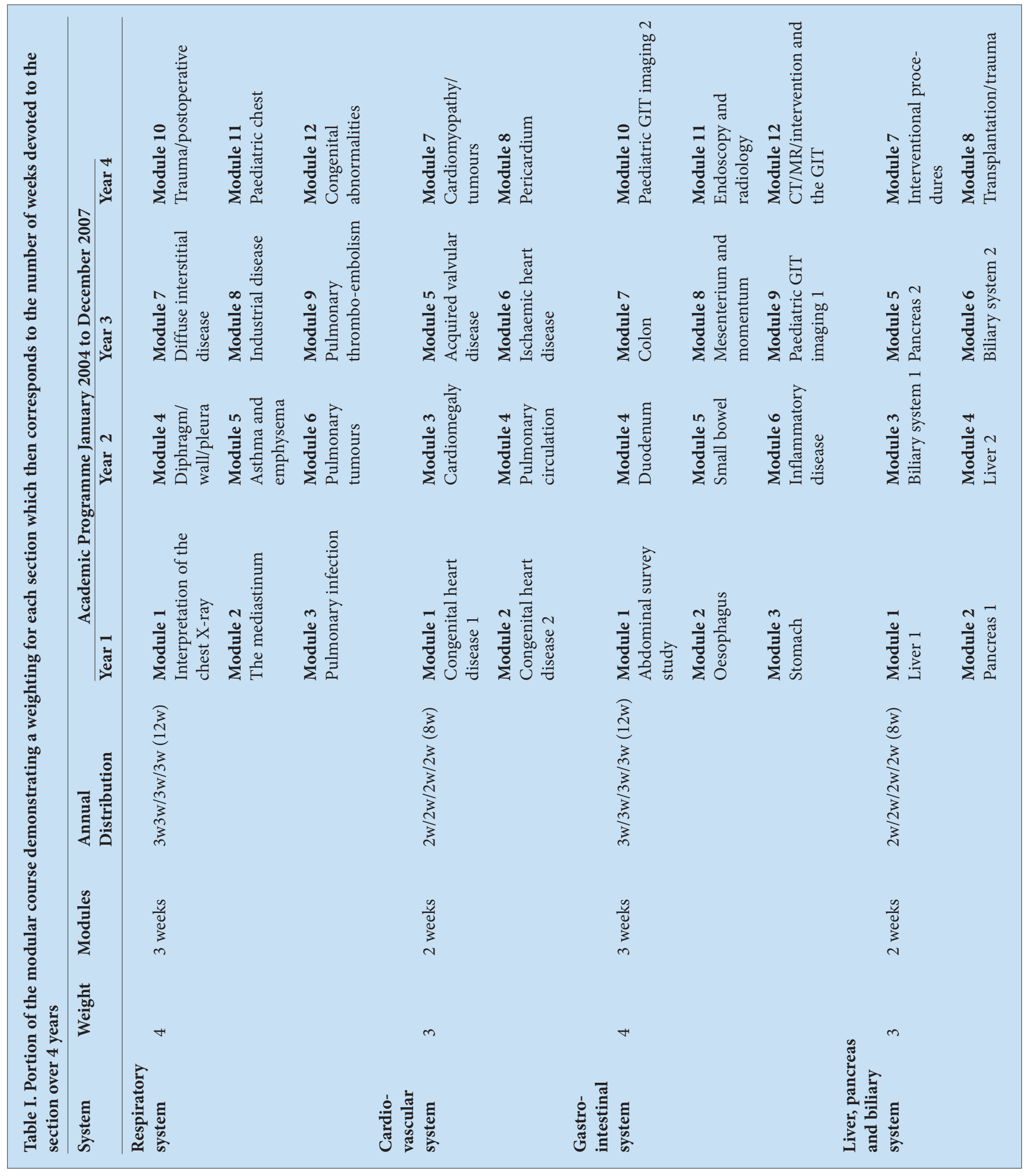


Table II. Example of detailed content of one module composed of between four and five 20-minute lectures for each module (on 1 day per week).

Year 1: 2004

Module 1

Metabolic bone disease 1

Consultant Lecture (Optional)

Lecture 1

Lecture 2

Lecture 3

Lecture 4

Module 2

Metabolic bone disease

Consultant Lecture (Optional)

Lecture 1

Lecture 2

Lecture 3

Lecture 4

Module 3

Bone Tumours 1

National Videoconference

Lecture 1

Lecture 2

Lecture 3

Lecture 4
Physiology of bone

Hyper/hypoparathyroidism

Osteoporosis/osteomalacia

Rickets

Conditions associated with increase in bone density

Diagnostic approach to the skeleton

Conditions associated with increase in bone density

Renal osteodystrophy/hyperphosphatasia

Scurvy/Cushings/juvenile osteoporosis

Conditions associated with decreased bone density

Diagnostic approach to bone tumours

Benign tumours

Cartilaginous tumours

Cystic mass lesions

Fibrous tumours currently in the 3rd year of the first cycle. Lectures of outstanding quality have been captured on digital disk as an integral component of the course. Table II is an example of individual lectures comprising modules 1 , 2 and 3 of the musculo-skeletal system for year one. Note that the number of modules is decided by weighting each section on a scale of 1 to 4 . The course then extends over 25 weeks each year or 100 weeks over the 4 -year period. This allows enough time for flexibility regarding holidays, revision and examination. A national videoconference would conclude a set of modules and we hope to have recognised experts in a specific field to present these lectures to all participants: academic, private, South African and African.
We would then extend an open invitation to our registrars to jump onto the 'merry-go-round' at any point and to jump off 4 years later to write an examination of international standard. There is one condition: the entire 4 years must be devoted to learning radiology. The ticket to the 4-year 'merry-go-round' is a valid primary examination from the College of Radiology or a recognised university.

\section{Jan W Lotz}

